\title{
Controlling Chemotherapy-Induced Nausea and Vomiting with Neurokinin-1 Receptor Antagonists in Patients on AC-Based Chemotherapy-Are We There Yet?
}

\author{
Kevin Yap ${ }^{1 *}$, Cassandra Leong ${ }^{2}$, Alexandre Chan ${ }^{2,3}$ \\ ${ }^{1}$ Institute of Digital Healthcare, University of Warwick, Coventry, UK; ${ }^{2}$ Department of Pharmacy, Faculty of Science, National Uni- \\ versity of Singapore, Singapore; ${ }^{3}$ Department of Pharmacy, National Cancer Centre Singapore, Singapore. \\ Email: "kevinyap.ehealth@gmail.com,k.yap@warwick.ac.uk
}

Received January $9^{\text {th }}, 2012$; revised February $6^{\text {th }}, 2012$; accepted February $16^{\text {th }}, 2012$

\begin{abstract}
Chemotherapy-induced nausea and vomiting (CINV) are distressing side effects of chemotherapy. Neurokinin-1 receptor antagonists (NK1-RAs) have been incorporated in the contemporary management of CINV. However, clinical studies on NK1-RAs have shown mixed results in reducing CINV risk. Most studies focused on the use of aprepitant (APR) and casopitant (CAS) in breast cancer patients receiving AC-type (doxorubicin and cyclophosphamide) chemotherapy. In this study, we compared the study design and clinical efficacies of these NK1-RAs in reducing CINV risk. Among the selected eight studies, 4 APR Randomized Controlled Trials (RCTs), 2 APR Observational Studies (OSs) and 2 CAS RCTs were identified. Patient-related characteristics such as the proportion of females $(60.0 \%-100.0 \%)$, age (46.5 - 59.5 years), histories of motion $(5.6 \%-47.0 \%$ in NK1-RA arms) and morning sicknesses $(14.2 \%-45.0 \%$ in NK1-RA arms) and types of antiemetic regimens; as well as chemotherapy-related characteristics such as the proportion of patients on AC chemotherapy (15.0\% - 100.0\%) varied greatly. In terms of efficacies, both APR and CAS improved overall CR and vomiting in majority of the studies. None of the studies, however, demonstrated that NK1-RA could provide adequate nausea control. To conclude, NK1-RAs are effective in improving vomiting and overall CR, but not useful in controlling nausea or attaining CC, the ideal CINV endpoint. A shift in paradigm is needed for future CINV research. As healthcare providers continue to strive for optimum CINV control in their patients, we hope this review can help them make better informed clinical decisions.
\end{abstract}

Keywords: AC; Anthracycline-Based Chemotherapy; Aprepitant; Breast Cancer; Casopitant; Chemotherapy-Induced Nausea; Vomiting; Neurokinin-1 Receptor Antagonists (NK1-RAs)

\section{Introduction}

Chemotherapy-induced nausea and vomiting (CINV) are distressing side effects of chemotherapy [1]. Despite conventional antiemetic management, $40 \%$ of patients on moderately emetogenic chemotherapies (MECs) continue to experience CINV $[2,3]$. In recent years, studies have identified a new class of drugs, the neurokinin-1 receptor antagonists (NK1-RAs), to be effective in controlling CINV [4]. Traditional MEC antiemetic regimens entail a 5hydroxytryptamine- 3 serotonin receptor antagonist $\left(5 \mathrm{HT}_{3^{-}}\right.$ RA) and corticosteroid, usually dexamethasone [5-7]. While $5 \mathrm{HT}_{3}$-RAs inhibit $5 \mathrm{HT}_{3}$ receptors in the chemoreceptor trigger zone [8] and corticosteroids could possibly prevent emesis by prostaglandin antagonism [9], NK1-RAs have an entirely different mechanism. As se-

*The authors have no conflicts of interest that are directly relevant to the content of this study. lective, high-affinity antagonists to NK1 receptors, they alleviate amounts of the emetic substance P [10], thus making them useful adjuvants in optimal CINV control.

Aprepitant (APR) was approved by the Food and Drug Administration (FDA) in 2003, while another NK1-RA, casopitant (CAS), has shown promising outcomes, with research progressing to Phase III trials [11,12]. As a result, the American Society of Clinical Oncology (ASCO), Multinational Association of Supportive Care in Cancer (MASCC), European Cancer Organisation (ECCO) and National Comprehensive Cancer Network (NCCN) have since incorporated the use of NK1-RAs in their antiemetic clinical practice guidelines [5-7].

Breast cancer, the most frequently occurring cancer (29.2\%) among females in Singapore [13] falls under the purview of these clinical updates. It is commonly treated with an anthracycline and cyclophosphamide-based (ACbased) regimen [14-16]. Although this regimen is tradi- 
tionally classified as a MEC, it demonstrates patterns similar to highly emetogenic chemotherapies (HECs), thus warranting the adjuvant use of NK1-RAs for optimal CINV control [5-7].

With the high incidence of breast cancer in Singapore, NK1-RAs can potentially revolutionize CINV control for a large proportion of patients. However, randomized, controlled trials (RCTs) that are used to substantiate these clinical guidelines [5-7] have had differing methodologies in terms of design-related, patient-related and chemotherapy-related characteristics. In addition, certain risk factors are known to predispose patients towards CINV. These differences may have considerably influenced the interpretation of NK1-RA efficacies in CINV control, making it difficult to attain a holistic view on the usefulness of NK1-RAs in various cancer populations on AC-based regimens. As such, we did an evidence-based evaluation of the current literature relating to NK1-RA efficacies in various AC-based populations, so that oncology healthcare professionals can make better informed clinical decisions as they strive for optimal CINV control in their patients. We also look at the progress of CINV research in the past year, and suggest several areas which may help optimize future CINV management.

\section{Methodology}

A literature search was conducted in June 2010 on PubMed and the abstract databases of ASCO, MASCC, ECCO and NCCN using a combination of the keywords "aprepitant", "casopitant", "NK-1 antagonists", "chemotherapy-induced nausea and vomiting", "AC chemotherapy”, "Adriamycin/doxorubicin”, "epirubicin”, "anthraxcycline", "cyclophosphamide", "breast cancer" and "moderately emetogenic". Studies included in our review were RCTs or observational studies (OSs) of patients greater than 18 years of age, and with a substantial proportion of breast cancer patients on AC-based chemotherapy. Papers and abstracts on the antiemetic activities of NK1-RAs in breast cancer patients on AC-based chemotherapy were identified. Exclusion criteria were non-English studies, animal studies, phase 0 studies, studies of NK1-RAs in post-operative vomiting, and those pertaining to other chemotherapy regimens. A total of 153 studies published between 2005 and 2010 were identified from our search, with 8 studies remaining after applying the inclusion and exclusion criteria. Of these, 4 RCTs and 2 OSs focused on aprepitant, while 2 RCTs investigated the efficacy of casopitant (Figure 1).

Comparison and contrast of these 8 studies were conducted through descriptive statistics. Extracted data were categorized into 1) design-related; 2) patient-related; 3) chemotherapy-related; and 4) CINV-related characteristics in Microsoft Excel 2007 for easy qualitative comparisons. Design-related characteristics encompassed how the studies were carried out (e.g. whether the study was a RCT or OS, unicentre or multicentre, national or international, and inclusion and exclusion criteria for the recruited patients). Patient-related characteristics were the number of patients, the percentage of females and patients on AC-based chemotherapy, as well as the proportion of patients with CINV risk factors (e.g. history of motion sickness, morning sickness and alcohol consumption). Chemotherapy-related characteristics included the emetogenicity of regimens and chemo-naivety. Lastly, CINV-related characteristics were the proportion of patients who experienced "no nausea" and "no vomiting" regardless of rescue medication use, or achieved complete response (CR, defined as no vomiting and no rescue medication use), complete protection ( $\mathrm{CP}$, defined as $\mathrm{CR}$ and no significant nausea) and complete/total control (CC/TC, defined as CR and no nausea). These endpoints were measured in the acute $(0-24$ hours after chemotherapy), delayed (24 - 120 hours after chemotherapy) and overall $(0-120$ hours after chemotherapy) phases. Subsequently, trends and differences in CINV-related characteristics among the studies, such as whether the NK1-RA was useful in controlling nausea and vomiting, were elucidated. It is important to note that the CINV data reported by Grote et al. [17] and Arpornwirat et al. [18] pertained to the entire study population, including non-AC-based patients. Data purely on AC-based pa-tients was not available from their respective publications. A comparison of statistical significance and absolute differences between the NK1-RA and control (CTL) arms among the RCTs was carried out. The absolute differences were used as comparative indicators of efficacy - a greater magnitude of absolute difference implied a more efficacious NK1-RA.

\section{Results and Discussion}

\subsection{Design-Related Characteristics of the Reviewed Studies}

\subsubsection{Study designs}

A total of 8 published studies were identified [11,12, 17-22] (Table 1). All 4 APR RCTs [12,19,21,22] and 2 CAS RCTs $[11,18]$ were prospective and double-blinded. Among the 4 APR RCTs, 2 were double-dummies $[19,21]$ and 3 were parallel groups $[12,19,21]$. Two APR RCTs were multinational $[12,21]$. Of these 2 APR RCTs, 1 was multicentered [19] and the other was unicentered [22]. Both the CAS RCTs were multinational $[11,18]$. Two open-labelled APR OSs were also identified [17,20].

\subsubsection{Inclusion and Exclusion Criteria}

Similar patient inclusion criteria were employed across the studies [11,12,17-22]. All studies required patients to 
Literature search of studies on aprepitant (APR)/casopitant (CAS) efficacies in CINV control

Sources: PubMed, American Society of Clinical Oncology (ASCO), Multinational Association of Supportive Care in Cancer (MASCC), European Cancer Organisation (ECCO) and National Comprehensive Cancer Network (NCCN) abstract databases

Keywords: aprepitant, casopitant, NK-1 receptor antagonists, chemotherapy-induced nausea and vomiting, AC chemotherapy, Adriamycin' doxorubicin, epirubicin, anthracycline, cyclophosphamide, breast cancer, moderately emetogenic

Inclusion criteria: randomized controlled trials (RCTs), observational studies (OSs), subjects above 18 years old consisting mainly of patients on AC-based chemotherapy

Exclusion criteria: non-English articles, animal and phase 0 studies, studies of NK1-RAs in post-operative vomiting and other chemotherapy regimens

Total number of studies identified from literature search: 153

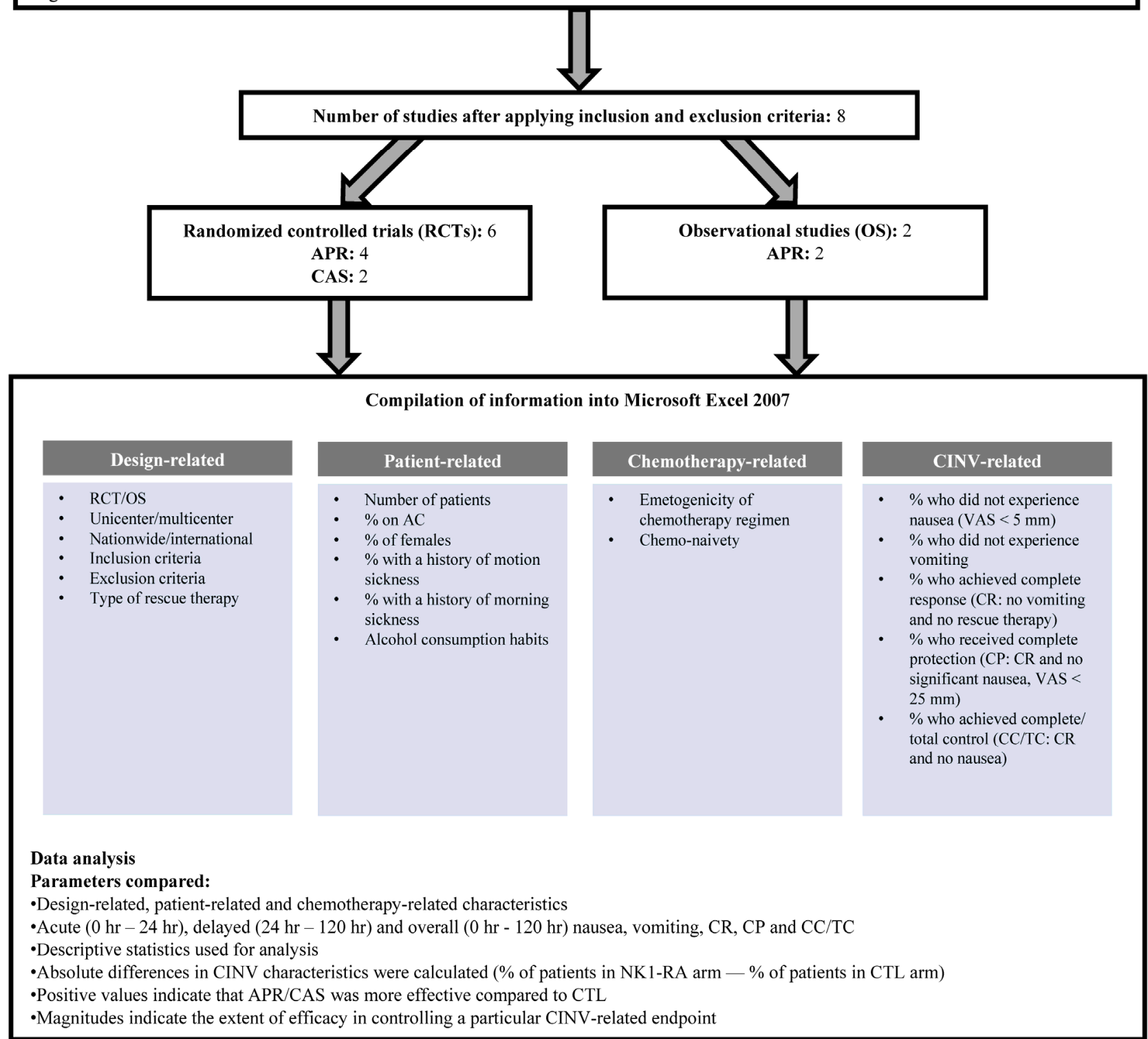

Figure 1. Flowchart of methodology. 


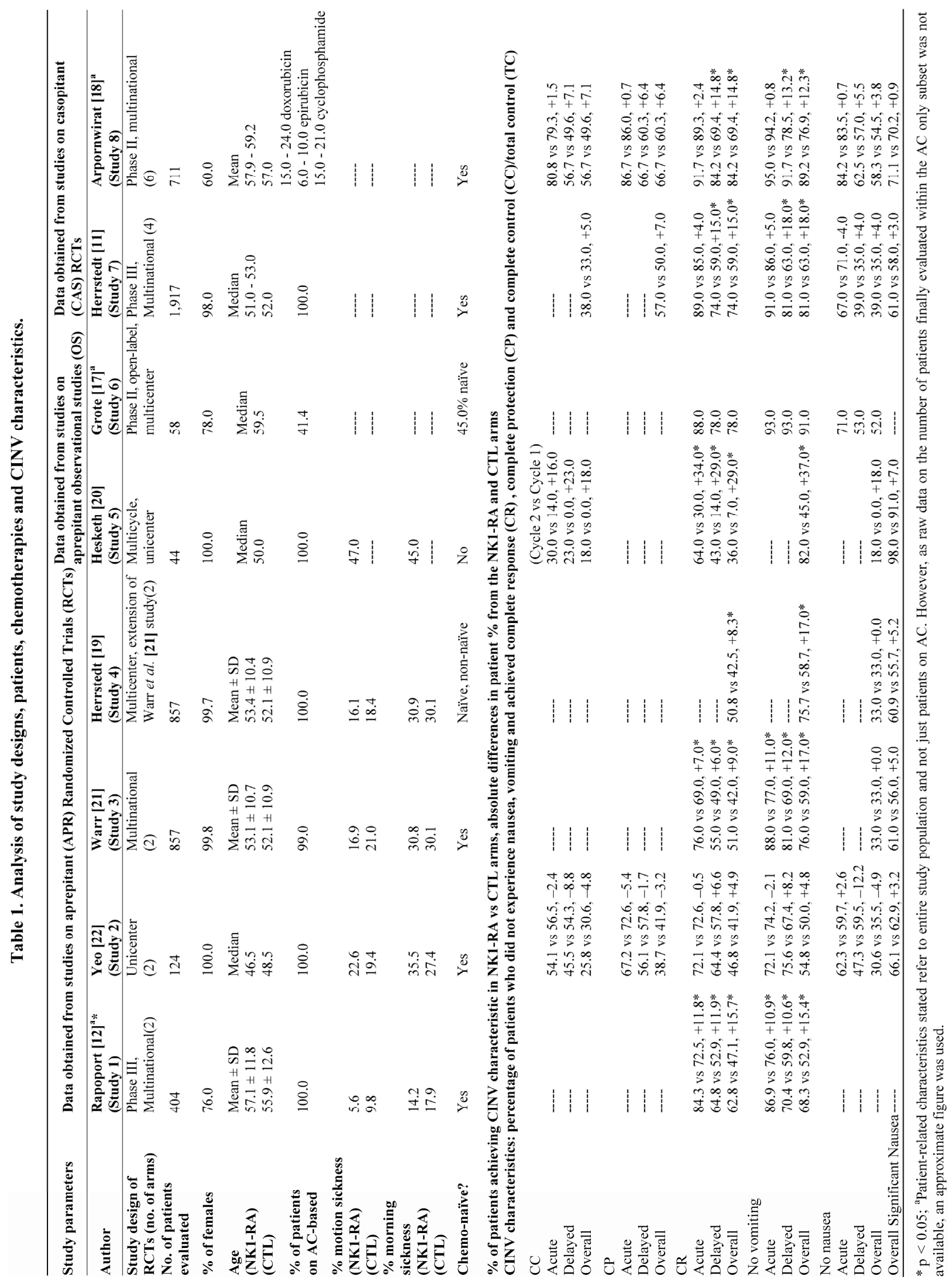


receive a course of MEC for the treatment of a solid malignant tumor. Four APR RCTs [12,19,21,22], 1 APR OS [20] and 1 CAS RCT [11] required patients to be on AC-based or AC-only chemotherapy. One CAS RCT and 1 APR OS included patients on other MECs such as fluorouracil, oxaliplatin [18], irinotecan [17,18], carboplatin, idarubicin, ifosfamide, mitoxantrone and $\leq 50 \mathrm{mg} / \mathrm{m}^{2}$ of cisplatin [17].

Three studies excluded patients receiving systemic corticosteroids $[12,18,21]$, while 3 studies excluded patients on drugs known to possess antiemetic activity such as lorazepam $[11,17,18]$. However, in the APR RCT by Warr et al. [21], patients were allowed to take single daily doses of lorazepam in the 48 hours preceding chemotherapy. Excluded patients in these studies were those with etiology known to increase CINV risk, such as active infections $[12,17,18,21,22]$, any uncontrolled disease $[12,17,18,21,22]$, symptomatic primary or metastatic central nervous system malignancy $[12,18,21]$, alcohol abuse, use of illicit drugs, mental incapacitation, significant emotional or psychiatric disorder or hypersensitivity to $5 \mathrm{HT}_{3}$-RAs or dexamethasone $[11,22]$. As the NK1RAs (e.g. aprepitant) were CYP3A4 substrates and inhibitors [23], two studies excluded patients concurrently on other CYP3A4 substrates $[11,18]$. Both studies excluded patients who received CYP3A4/5 inducers within 14 days or CYP3A4/5 inhibitors 2 - 14 days before treatment. Examples of CYP3A4 substrates that fitted this exclusion criteria were astemizole, pimozide, terfenadine, repaglinide or torsemide [11].

To minimize the contribution of anticipatory nausea and vomiting to CINV, 6 studies excluded patients who experienced nausea or vomiting in the 24 hours preceding chemotherapy $[12,17,18,20-22]$. Both APR OSs $[17,20]$ specifically stated the use of the National Cancer Institute Common Toxicity Criteria (Version 3) [24], which was not mentioned in the other 4 studies [12,18,21,22]. The study by Hesketh et al. [20] excluded patients who experienced nausea greater or equal to Grade 1, while that of Grote et al. [17] only excluded patients who had experienced nausea corresponding to Grade 2 or 3.

\subsubsection{Antiemetic Regimens}

A variety of antiemetic regimens were employed (Table 2). All 4 APR RCTs used the recommended dosing regimen of aprepitant $125 \mathrm{mg}$, ondansetron $16 \mathrm{mg}$ and dexamethasone $12 \mathrm{mg}$ on Day 1 and aprepitant $80 \mathrm{mg}$ on Days 2 - 3 in the APR arm [5]. In the CTL arm, patients were administered ondansetron $16 \mathrm{mg}$ and dexamethasone $20 \mathrm{mg}$ on Day 1 in the CTL arm as well as additional prophylaxis of ondansetron $16 \mathrm{mg}$ on Days 2 and 3 $[12,19,21,22]$. The $5 \mathrm{HT}_{3}$-RAs administered differed between the APR OSs and other studies. While only ondan- setron was administered in both APR and CAS RCTs [11,12,18,19,21,22], the OS by Hesketh et al. [20] (study 5) also included patients who received granisetron and dolasetron. All patients in the OS by Grote et al. [17] (study 6) were administered palonosetron. Differences in the $5 \mathrm{HT}_{3}$-RA used may have masked the efficacy of the NK1-RA in CINV control. For example, in a doubleblinded, randomized, phase II trial, palonosetron was found to be more efficacious than ondansetron in controlling CINV [25]. In addition, varying dosages and administration routes were used among studies. The APR RCTs used only oral (PO) administration, while APR OSs and CAS RCTs used both PO and intravenous (IV) administration routes. Although aprepitant dosages were consistent across APR RCTs [12,19,21,22] and OSs [17, 20], casopitant dosages in the various arms of the CAS RCTs ranged from 50 - $150 \mathrm{mg}$ PO $[11,18]$.

Two APR RCTs and 1 APR OS stated the rescue therapy permitted, which included metoclopramide [22], prochlorperazine, $5 \mathrm{HT}_{3}$-RAs [20], phenothiazines, butyrophenones, benzodiazepines, benzamides, corticosteroids and domperidone [21].

\subsection{Patient-Related Characteristics of the Reviewed Studies}

\subsubsection{Number of Patients}

The APR RCTs $[12,19,21,22]$ generally evaluated a smaller number of patients on AC-based chemotherapies (124 - 857 patients) than the CAS RCTs [11,18] (107 - 1917 patients). The total number of patients across the APR RCTs (1385 patients) was lower than the combined total of patients in the CAS RCTs (2024 patients). Similarly, the OSs $[17,20]$ had much smaller populations on ACbased chemotherapy (24 - 44 patients).

\subsubsection{Gender}

Females are associated with a greater risk of CINV. A prospective study by Hesketh et al. [26] evaluating the association between risk factors and CR from the data of 1043 patients in two identically designed phase III trials found that males $(p=0.023)$, but not females, was significantly associated with $\mathrm{CR}$. This was also observed in our reviewed studies - a higher percentage of females tended towards poorer CINV control. The APR RCTs $(76 \%-100 \%)[12,19,21,22]$ and OSs (78\% - 100\%) [17, $20]$ had a greater proportion of females than the CAS RCTs $(60 \%$ - 98\%) [11,18].

\subsubsection{Age}

Age is known to correlate inversely with CINV risk. The same study by Hesketh et al. [26] found a significant association between patients older than 65 years and $\mathrm{CR}$ $(\mathrm{p}=0.021)$. A similar phase III trial of patients on AC by 
in Patients on AC-Based Chemotherapy-Are We There Yet?

Table 2. Antiemetic regimens in the reviewed studies.

\begin{tabular}{|c|c|c|c|c|c|c|c|}
\hline \multirow{2}{*}{ Study } & \multirow{2}{*}{ Regimen } & \multicolumn{3}{|c|}{ Day 1 (Acute) } & \multicolumn{2}{|c|}{ Days 2 - 3 (Delayed) } & \multirow[b]{2}{*}{ DEX } \\
\hline & & NK1-RA & 5HT3-RA & DEX & NK1-RA & 5HT3-RA & \\
\hline \multirow{3}{*}{$\begin{array}{l}\text { Rapoport [12], } \\
\text { Herrstedt [19], } \\
\text { Warr [21], } \\
\text { Yeo [22] }\end{array}$} & APR & APR $125 \mathrm{mg}$ & OND $16 \mathrm{mg}$ & DEX $12 \mathrm{mg}$ & APR $80 \mathrm{mg}$ & - & - \\
\hline & CTL & - & OND $16 \mathrm{mg}$ & DEX $20 \mathrm{mg}$ & - & OND $16 \mathrm{mg}$ & - \\
\hline & Phase I & - & $\begin{array}{l}\text { OND } 24 \mathrm{mg} / 8 \mathrm{mg} \text { IV } \\
\text { DOL } 100 \mathrm{mg} / 100 \mathrm{mg} \text { IV } \\
\text { GRAN } 2 \mathrm{mg} / 1 \mathrm{mg} \text { IV }\end{array}$ & $\begin{array}{l}\text { DEX } 8 \text { - } 10 \mathrm{mg} / \\
8-10 \mathrm{mg} \text { IV }\end{array}$ & - & - & DEX $8 \mathrm{mg}$ \\
\hline Hesketh [20] & Phase II & APR $125 \mathrm{mg}$ & $\begin{array}{l}\text { OND } 24 \mathrm{mg} / 8 \mathrm{mg} \text { IV } \\
\text { DOL } 100 \mathrm{mg} / 100 \mathrm{mg} \text { IV } \\
\text { GRAN } 2 \mathrm{mg} / 1 \mathrm{mg} \text { IV }\end{array}$ & $\begin{array}{l}\text { DEX } 8-10 \mathrm{mg} / \\
8-10 \mathrm{mg} \text { IV }\end{array}$ & APR $80 \mathrm{mg}$ & - & DEX $4 \mathrm{mg}$ \\
\hline Grote [17] & APR & APR125 mg & PAL $0.25 \mathrm{mg}$ IV & DEX $12 \mathrm{mg}$ & APR $80 \mathrm{mg}$ & - & DEX $8 \mathrm{mg}$ \\
\hline \multirow[t]{2}{*}{ Herrstedt [11] } & CAS & $\begin{array}{l}\text { CAS } 150 \mathrm{mg} \\
\text { CAS } 150 \mathrm{mg} \\
\text { CAS } 90 \mathrm{mg} \text { IV }\end{array}$ & OND $16 \mathrm{mg}$ & DEX 8 mg IV & $\begin{array}{l}- \\
\text { CAS } 50 \mathrm{mg} \\
\text { CAS } 50 \mathrm{mg}\end{array}$ & OND $16 \mathrm{mg}$ & $\begin{array}{l}- \\
- \\
-\end{array}$ \\
\hline & CTL & - & OND $16 \mathrm{mg}$ & DEX $8 \mathrm{mg}$ IV & - & OND $16 \mathrm{mg}$ & - \\
\hline \multirow[t]{2}{*}{ Arpornwirat [18] } & CAS & $\begin{array}{l}\text { CAS } 50 \mathrm{mg} \\
\text { CAS } 100 \mathrm{mg} \\
\text { CAS } 150 \mathrm{mg}\end{array}$ & OND $16 \mathrm{mg}$ & DEX 8 mg IV & $\begin{array}{l}\text { CAS } 50 \mathrm{mg} \\
\text { CAS } 100 \mathrm{mg} \\
\text { CAS } 150 \mathrm{mg}\end{array}$ & OND $16 \mathrm{mg}$ & $\begin{array}{l}- \\
- \\
-\end{array}$ \\
\hline & CTL & - & OND $16 \mathrm{mg}$ & DEX 8 mg IV & - & OND $16 \mathrm{mg}$ & - \\
\hline
\end{tabular}

NK1-RA: neurokinin-1 receptor antagonist, 5HT3-RA: 5-hydroxytryptamine-3 receptor antagonist, APR: aprepitant, CAS: casopitant, DEX: dexamethasone, OND: ondansetron, GRAN: granisetron, DOL: dolasetron, PAL: palonosetron, PO: oral, IV: intravenous. All administration routes were oral (PO) unless otherwise stated.

Warr et al. [27] also noted a significant relationship between patients older than 55 years and CR $(p=0.006)$. A trend between age and CINV was seen among the data as well. Four studies reported mean age [12,18,19,21], while the other 4 studies reported median age $[11,17,20$, 22]. Studies with smaller populations used median age indicators, while larger studies used mean age. In terms of mean age, the CAS RCT conducted by Arpornwirat et al. [18] (study 8) had older patients (57.9 - 59.2 years across 3 CAS arms and 57.0 years in CTL arm) than the 3 APR RCTs [12,19,21] (52.1 - 57.1 years across all arms and studies). Incidentally, the study by Arpornwirat et al. [18] (84.2\%) demonstrated better overall CR in the NK1-RA arm compared to the 3 APR RCTs $[12,19,21]$ $(50.8 \%-62.8 \%)$. In terms of median age, the CAS RCT conducted by Herrstedt et al. [11] (study 7) (51.0 - 53.0 years) had older patients than the APR RCT by Yeo et al. [22] (study 3) (46.5 - 48.5 years). Likewise, Herrstedt et al. [11] $(74.0 \%)$ also reported higher overall CR in the NK1-RA arm compared to the study by Yeo et al. [22] (46.8\%). The APR OSs had varying median ages ranging from 50.0 - 59.5 years $[17,20]$.

\subsubsection{History of Motion Sickness}

Patients with a history of motion sickness have been reported to be more prone to CINV [28]. However, the study by Warr et al. [27] found this risk factor to be insignificant ( $\mathrm{p}>0.05)$. All APR RCTs [12,19,21,22] reported this statistic while none of the CAS RCTs did so. The smallest proportions of patients with a history of motion sickness in the NK1-RA and CTL arms were $5.6 \%$ and $9.8 \%$ respectively in the study conducted by Rapoport et al. [12] (study 1); while the largest proportions were $22.6 \%$ and $19.4 \%$ in the study by Yeo et al. [22] (study 3). Interestingly, there seemed to be a considerable association between motion sickness history and CR, as Rapoport et al. [12] reported a greater overall CR $(62.8 \%)$ in the NK1-RA arm than Yeo et al. [22] (46.8\%). In the APR OS conducted by Hesketh and colleagues [20] (study 5), $47.0 \%$ of patients had a history of motion sickness. Correspondingly, the $\mathrm{CR}$ obtained was also much lower $(36.0 \%)$ than the RCTs.

\subsubsection{History of Morning Sickness}

A history of morning sickness has been suggested to increase CINV risk as well [28]. Findings from Warr et al. [27] further support this, with a significant association between morning sickness history and CINV ( $p=0.0007)$. In our review, the same APR RCTs reported the patient proportion with a history of motion sickness [12,19,21, 22]. Among the APR RCTs, the smallest proportions in the NK1-RA and CTL arms were $14.2 \%$ and $17.9 \%$ reported by Rapoport et al. [12] (study 1), and the greatest proportions were $35.5 \%$ and $27.4 \%$ in the study by Yeo et al. [22] (study 3). Hesketh and colleagues [20] (study 5) reported a relatively high proportion (45.0\%) of patients with a history of morning sickness. Again, the level of CINV control corresponded to the extent of this risk factor. The Rapoport study [12] (62.8\%) had a high- 
er overall CR in the NK1-RA arms than the Yeo [22] $(46.8 \%)$ and Hesketh [20] (36.0\%) studies. All studies only considered females who had been pregnant for the derivation of these figures.

\subsubsection{History of Alcohol Consumption}

Patients who do not consume alcohol have a higher predisposition towards CINV [28]. While an optimal alcohol consumption level has yet to be determined, both Hesketh et al. [26] $(\mathrm{p}=0.027)$ and Warr et al. [27] $(\mathrm{p}=$ 0.0048 ) found that consumption of $\geq 5$ drinks a day significantly improved CINV control, while non-drinkers experienced the worst CINV. The APR OS by Hesketh and colleagues [20] (study 5) classified alcohol consumption into 3 categories: non-user $(35.0 \%)$, social drinkers (less than 7 drinks a week, 50.0\%) and chronic drinkers (more than 1 drink a day, 15.0\%). As it was the only study to describe alcohol consumption habits, it was not possible to compare the association between alcohol consumption history and CINV.

\subsection{Chemotherapy-Related Characteristics of the Reviewed Studies}

\subsubsection{Chemotherapy Regimen}

The emetogenicity of the regimen increases CINV risk [28]. As AC-based regimens exhibit HEC patterns, populations with a greater proportion of patients on AC-based regimens rather than less emetogenic regimens may have poorer CINV control. The percentage of patients on ACbased regimens among studies ranged from $41.4 \%-100.0 \%$ [11,12,17-22]. All APR RCTs had 100\% AC-based sample populations $[12,19,21,22]$, although the administered doses of anthracycline and cyclophosphamide differed. This may again have affected the CINV risk of patients. For example, patients in the RCT conducted by Yeo et al. [22] (study 3) (doxorubicin $60 \mathrm{mg} / \mathrm{m}^{2}$ and cyclophosphamide $600 \mathrm{mg} / \mathrm{m}^{2}$ ) may have had an increased predisposition towards CINV compared to the OS by Hesketh et al. [20] (study 5) (doxorubicin $<60 \mathrm{mg} / \mathrm{m}^{2}$ and cyclophosphamide $\left.>500 \mathrm{mg} / \mathrm{m}^{2}\right)$ or the RCT by Rapoport et al. [12] (study 1) (any dose of doxorubicin/epirubicin and IV cyclophosphamide $<1500 \mathrm{mg} / \mathrm{m}^{2}$ ). The study by Yeo et al. [22] did indeed exhibit a lower overall CR (46.8\%) in the NK1-RA arm than the study by Rapoport et al. [12] (62.8\%).

In contrast, the proportion of patients on AC-based regimens in the CAS RCTs were lower and ranged from $15 \%-100 \%[11,18]$. As no information on the percentage of patients on AC in the study by Arpornwirat et al. [18] was available, the estimated figure of $15 \%$ was derived based on a minimum proportion of patients on cyclophosphamide (15\%), doxorubicin $(15 \%-24 \%)$ and epirubicin $(6 \%-10 \%)$. As expected, Arpornwirat et al. [18] reported a higher overall CR in the NK1-RA arm (84.2\%) compared to Herrstedt et al. [11] (74.0\%).

In the CAS RCT by Arpornwirat et al. [18], a majority $(50 \%-56 \%)$ of patients were on the less emetogenic carboplatin regimen compared to doxorubicin $(15 \%-24 \%)$, epirubicin $(6 \%-10 \%)$ and cyclophosphamide $(15 \%-21 \%)$. Although majority of the patients in the Grote OS [17] were on AC-based regimens (41.4\%), a smaller proportion was on a carboplatin/paclitaxel-based regimen (22.4\%). This may have decreased the population's collective risk towards CINV, as shown by the higher overall $\mathrm{CR}$ values in the NK1-RA arm as compared to similar studies with $100 \%$ AC populations.

\subsubsection{Chemo-Naivety}

As patients with a history of CINV are more likely to experience CINV in subsequent cycles, non-naive samples may have a higher risk of CINV. All APR and CAS RCTs included only chemo-naive patients in their samples $[11,12,18,19,21,22]$.

An extension study conducted by Herrstedt et al. [19] which followed up on patients who had completed Cycle 1 of chemotherapy in the RCT by Warr and colleagues [21], and were in their subsequent cycles, gave certain insights regarding chemo-naivety and CINV control. In this study, the proportion of patients who attained $\mathrm{CR}$ steadily decreased in the APR versus CTL arms; from $50.8 \%$ versus $42.5 \%$ in Cycle 1 , to $40.9 \%$ versus $30.7 \%$ in Cycle 2, 37.9\% versus $26.3 \%$ in Cycle 3 and $34.5 \%$ versus $23.9 \%$ in Cycle 4 . The treatment advantage of APR also increased slightly over the cycles from $8.3 \%$ in Cycle 1 to $14.4 \%$ in Cycle 2, $14.8 \%$ in Cycle 3 and $16.6 \%$ in Cycle 4.

The APR OS by Grote et al. [17] surveyed a smaller proportion of non-naive patients $(45.0 \%)$ compared to the multi-cyclic OS by Hesketh and colleagues [20] (study 5) (100.0\%) which could have accounted for the much higher overall CR of $78.0 \%$ compared to $36.0 \%$.

\subsection{CINV-Related Characteristics of the Reviewed Studies}

\subsubsection{Assessment of Nausea and Vomiting}

All studies required patients to keep a daily diary for 5 days following chemotherapy [11,12,17-22]. The diary documented the date and time of emetic episodes, rescue therapy use, and daily nausea ratings [11,12,17-22]. Three studies also performed follow ups, with a research nurse telephoning patients to assist with diary completion and to promote compliance with the antiemetic regimens [18, $20,22]$. This method of follow up was not reported in the other 5 studies [11,12,17,19,21].

Both APR and CAS RCTs evaluated nausea on a 100 $\mathrm{mm}$ visual analogue scale (VAS) [11,12,18,19,21,22]. 
Patients recorded their nausea experiences using the VAS with the understanding that 0 and 100 represented "no nausea" and "the worst it could get". For the purpose of analysis, "no nausea" was defined as VAS $<5 \mathrm{~mm}$, while "no significant nausea" was defined as VAS $<25 \mathrm{~mm}$. On the other hand, a 4-point categorical scale was used to evaluate nausea in the APR OSs by Hesketh et al. [20] (study 5) and Grote et al. [17] (study 6). The relationship between the scale and nausea status was not mentioned in the former study. However, in the latter study, the numbers 0 - 3 corresponded to nausea severities of "none", "mild", "moderate" and "severe" respectively. Only patients who ranked their nausea as 0 on the 4-point scale were considered to have had "no nausea" [17].

Although the definition of "no vomiting" as the lack of an emetic episode was universal among all the studies $[11,12,17-22]$, the frequency of an emetic episode differed in 2 studies. While both the Rapoport [12] and Hesketh [20] studies considered retching and vomiting to contribute to an emetic episode, Rapoport and colleagues considered retches occurring a minute apart as separate emetic episodes, in contrast to Hesketh and colleagues who considered one to five retches occurring within a 5-minute period as an emetic episode. The other 6 studies did not provide any details about this parameter [11,17-19, 21,22].

The indicators of CINV used to gauge NK1-RA efficacy were quite consistent across the studies. The most basic indicators were the proportions of patients who experienced "no vomiting", "no nausea" (VAS $<5 \mathrm{~mm}$ ) and "no significant nausea" (VAS $<25 \mathrm{~mm}$ ). Studies also used more comprehensive indicators (or endpoints) which took into account the antiemetic effects, specifically the proportion of patients with complete response (CR, i.e. "no vomiting" and no rescue medications) [11,12,17-22], complete protection $(\mathrm{CP}$, i.e. $\mathrm{CR}$ and "no significant nausea") $[11,18,20,22]$ and complete/total control $(\mathrm{CC} /$ $\mathrm{TC}$, i.e. CR and "no nausea") $[11,18,20,22]$. These indicators/endpoints were measured during the acute $(0-24$ hours after chemotherapy), delayed (24 - 120 hours after chemotherapy) and overall phases (0 - 120 hours after chemotherapy).

\subsubsection{No Vomiting}

All 8 studies reported the "no vomiting" parameter [11, $12,17-22]$. Both APR $(+15.4 \%$ to $+17.0 \%, \mathrm{p}<0.10)$ $[12,19,21]$ and CAS RCTs $(+12.3 \%$ to $+18.0 \%, \mathrm{p}<0.10)$ $[11,18]$, with the exception of the APR RCT by Yeo et al. [22] (study 2), showed significantly improved absolute differences in the overall phases. These APR RCTs [12, $19,21]$ also showed significant improvements in the acute $(+10.9 \%$ to $+11.0 \%, \mathrm{p}<0.10)$ and delayed $(+10.6 \%$ to $+12.0 \%, \mathrm{p}<0.10)$ phases. In contrast, CAS $[11,18]$ was useful in the delayed $(+13.2 \%$ to $+18.0 \%, \mathrm{p}<0.03)$, but not acute $(+0.8 \%$ to $+5.0 \%, \mathrm{p}>0.05)$ phase. The APR RCT by Yeo et al. [22] (study 2) was the sole study where absolute differences in the overall $(+4.8 \%)$, acute $(-2.1 \%)$ and delayed $(+8.2 \%)$ phases were insignificant.

The "no vomiting" incidence in the APR OSs greatly exceeded that of the APR RCTs. For example, the study by Grote et al. [17] had relatively higher "no vomiting" rates in the overall $(91.0 \%)$, acute $(93.0 \%)$ and delayed (93.0\%) phases. In the APR OS by Hesketh et al. [20], in which patients were given aprepitant only in Cycle 2, significant improvement in "no vomiting" was observed between Cycle 2 and 1 in the overall ( $82.0 \%$ vs $45.0 \%$, p $<0.02$ ) phase.

\subsubsection{Complete Response}

As "no vomiting" is a component of CR, similar trends and differences were expected and observed for CR. All 8 studies reported CR values [11,12,17-22]. Only 1 study did not report CR for all 3 phases [19]. Again, with the exception of the study by Yeo et al. [22], both aprepitant $(+9.0 \%$ to $+15.7 \%, \mathrm{p}<0.015)[12,19,21]$ and casopitant $(+14.8 \%$ to $+15.0 \%, \mathrm{p}<0.0127)[11,18]$ significantly improved overall CR. Aprepitant also improved both acute $(+7.0 \%$ to $+11.8 \%, \mathrm{p}<0.03)$ and delayed $(+6.0 \%$ to $+11.9 \%, \mathrm{p}<0.06) \mathrm{CR}[12,19,21]$, while casopitant improved delayed $(+14.8 \%$ to $+15.0 \%, \mathrm{p}<0.0127)$ but not acute $(+2.4 \%$ to $+4.0 \%, \mathrm{p}>0.05) \mathrm{CR}[11,18]$. The APR RCT by Yeo et al. [22] reported very low and insignificant improvements in the overall $(+4.9 \%)$, acute $(-0.5 \%)$ and delayed $(+6.6 \%) \mathrm{CR}$.

\subsubsection{No Nausea}

Three APR RCTs $[19,21,22]$ and 2 CAS RCTs $[11,18]$ reported overall "no nausea". When comparing absolute differences, both the inclusion of aprepitant $(-4.9 \%$ to $0.0 \%)$ and casopitant $(+3.8 \%$ to $+4.0 \%)$ did not result in a significant increase $(\mathrm{p}>0.05)$ in overall nausea control. Casopitant $(+4.0 \%$ to $+5.5 \%)$ was useful in controlling delayed nausea. However, aprepitant $(+3.2 \%$ to $+5.2 \%)$ and casopitant $(+0.9 \%$ to $+3.0 \%)$ did not provide any significant benefit in controlling significant nausea.

\subsubsection{Complete Control}

Four studies reported overall CC [11,18,20,22], of which 3 studies $[18,20,22]$ reported acute and delayed CC statistics as well. Although overall CC improved in the 2 CAS RCTs $(+5.0 \%$ and $+7.1 \%$ respectively, $p>0.05)$ $[11,18]$, this was not statistically significant. The APR RCT by Yeo et al. [22] (study 2) actually showed a reduction in the proportion of patients who achieved $\mathrm{CC}$ in the APR compared to the CTL arm in the overall $(-4.8 \%)$, acute $(-2.4 \%)$ and delayed $(-8.8 \%)$ phases. 
The NK1-RAs did not significantly improve acute and delayed $\mathrm{CC}$ either. The absolute differences in overall $(+5.0 \%$ to $+7.1 \%)$, acute $(+1.5 \%)$ and delayed $(+7.1 \%)$ $\mathrm{CC}$ in the CAS RCTs $[11,18]$ were greater than the overall $(-4.8 \%)$, acute $(-2.4 \%)$ and delayed $(-8.8 \%) \mathrm{CC}$ in the APR RCT conducted by Yeo and colleagues [22]. In addition, the CAS RCTs by Herrstedt et al. [11] and Arpornwirat et al. [18] had greater overall (CAS: $38.0 \%$ 56.7\%, CTL: $33.0 \%$ - 49.6\%), acute (CAS: $80.8 \%$, CTL: $79.3 \%$ ) and delayed (CAS: $56.7 \%$, CTL: $49.6 \%$ ) CC values than the APR RCT by Yeo and colleagues [22] in the overall (APR: $25.8 \%$, CTL: $30.6 \%$ ), acute (APR: $54.1 \%$, CTL: $56.5 \%$ ) and delayed (APR: $45.5 \%$, CTL: $54.3 \%$ ) phases.

A significant improvement was noted in the multi-cyclic OS by Hesketh and colleagues [20] (study 5), where overall CC improved from $0.0 \%$ in Cycle $1 \%$ to $18.0 \%$ in Cycle 2. All comparisons made between NK1-RA and CTL arms were in Cycle 1 of chemotherapy except in the Hesketh OS (study 5) [11,18,20,22].

\subsubsection{Complete Protection}

The same 3 RCTs reported overall CP values [11,18,22]. Two studies also reported acute and delayed $\mathrm{CP}$ values $[18,22]$. The improvements in overall, acute and delayed CP were statistically insignificant $(\mathrm{p}>0.05)[11,18,22]$. However improvements in the overall $(+6.4 \%$ to $+7.0 \%)$, acute $(+0.7 \%)$ and delayed $(+6.4 \%) \mathrm{CP}$ were greater in the CAS RCT compared to the overall $(-3.2 \%)$, acute $(-5.4 \%)$ and delayed $(-1.7 \%) \mathrm{CP}$ of the APR RCT.

The CAS RCT by Arpornwirat et al. [18] had higher overall (CAS: $66.7 \%$, CTL: $60.3 \%$ ), acute (CAS: $86.7 \%$, CTL: $86.0 \%$ ) and delayed (CAS: 66.7\%, CTL: 60.3\%) CP than the overall (APR: $38.7 \%$, CTL: $41.9 \%$ ), acute (APR: $67.2 \%$, CTL: $72.6 \%$ ) and delayed (APR: $56.1 \%$, CTL: $57.8 \%$ ) CP values in the APR RCT by Yeo et al. [22], which could have been due to a lower patient propensity to experience CINV.

\subsection{Implications to CINV Control}

The analysis revealed a myriad of differences in designrelated, patient-related and chemotherapy-related characteristics. In terms of study design, APR studies had smaller sample populations, which may have compromised the study's accuracy in predicting NK1-RA efficacy. Both APR and CAS had varying sets of inclusion and exclusion criteria, but generally, the same level of stringency was maintained in all studies.

The level of CINV experienced seemed to correspond to the presence of risk factors such as the female gender and age. APR studies were more representative of a typical breast cancer population on $\mathrm{AC}$ due to their higher proportion of females and hence demonstrated poorer
CINV control. The APR studies also had a relatively younger population which could have again accounted for the poorer CINV control in APR studies compared to CAS studies. In addition, not all studies had 100\% AC sample populations, while others had a substantial proportion on less emetogenic regimens $[17,18]$. These factors did seem to reduce a patients' risk of CINV, and as such these studies were less representative of the AC population.

One of the most striking differences across studies was the proportion of patients who had motion and morning sickness risk factors. The range of patient proportions that had a history of motion sickness $(5.6 \%-47.0 \%)$ and morning sickness $(14.2 \%-45.0 \%)$ across studies was very wide. Another difference was the varying doses and types of antiemetics used which may have affected the efficacies of the antiemetic regimens. For example, palonosetron, a second generation $5 \mathrm{HT}_{3}-\mathrm{RA}$, was shown to be superior to ondansetron in preventing CINV [25], therefore patients on palonosetron would probably have better protection against CINV.

In addition to study designs, patient-related and chemotherapy-related factors previously analyzed, other factors unique to each study may have influenced CINV-related characteristics. For example, Yeo and colleagues [22] proposed that the insignificant improvements in CR rates in their RCT could have possibly resulted from a smaller proportion of patients taking rescue medications in the APR arm (18.0\%) compared to the CTL arm (32.0\%). As CR comprises both parameters of "no vomiting" and "no rescue medication use", this may have contributed to the equivocal CR rates between arms. Since aprepitant is both a substrate and moderate inhibitor of CYP3A4 [29, $30]$, genetic differences [29,31] may have accounted for the varying aprepitant efficacies between this study, which comprised of $100 \%$ ethnic Chinese patients, and similar studies which sampled a large proportion of Caucasians [22].

The NK1-RAs have demonstrated significant improvements in overall CR. Overall CR was the most comprehensive indicator of NK1-RA efficacy in emetic control as it took into account both no vomiting and rescue medication use, which explains why CR was uniformly chosen as an endpoint in all studies. However, when the incidences of nausea and vomiting were evaluated independently, it was alarming to realize that NK1-RAs did not demonstrate any statistical and clinically-important benefits in terms of nausea control. This can be easily overlooked as most of the studies utilized summative endpoints such as "overall CR" to demonstrate the benefits of NK1-RAs. However, it is important to note that this endpoint does not account for the incidence of nausea, a side-effect that is clinically-important and lacks attention. 
Future studies should utilize more robust endpoints (such as $\mathrm{CC}$ or $\mathrm{CP}$ ), and investigate into agents that can provide protection to patients from chemotherapy-induced nausea.

Although both aprepitant and casopitant are useful in CINV control, casopitant was withdrawn in October 2009 as GlaxoSmithKline assessed that further safety data, as requested by the US Regulatory Authority, would need additional time to be produced [32].

\section{Limitations of Current Study}

As our literature search was restricted to English studies, useful additional data may have been excluded. The available clinical data were few in number, which made it more difficult to compare data and depict trends.

In the evaluation of data, only qualitative comparisons were conducted. While the use of quantitative statistics may have presented a more accurate picture of NK1-RA efficacies in AC-based populations, the complex nature of differences in design-related, patient-related, chemotherapy-related and CINV-related characteristics made it unfeasible. As such, rather than making a comparison between the efficacies of the two drugs, this study aimed to elucidate the differences amongst these studies which may have caused these discrepancies.

Not all risk factors and their impact on CINV endpoints could be evaluated as this information was not available. The most readily available information were factors such as age and gender. However, only 5 studies reported other known risk factors such as the percentage of patients who had a history of motion sickness or pregnancy-induced nausea and vomiting [12,19-22]. Only one study reported the alcohol consumption habits of the patients [20].

While it would have been useful to evaluate the influence of patient and chemotherapy-related factors in CC and $\mathrm{CP}$, we were unable to do so due to varying contexts and designs of the 4 trials which reported these parameters $[11,18,20,22]$. Furthermore, the study by Yeo et al. [22] was actually anomalous among the rest of the APR RCTs as the APR arm performed worse than the CTL arm. Thus, such a comparison would have been inappropriate.

\section{CINV Control with NK1-RAs-Are We There Yet?}

This review has demonstrated that the NK1-RAs have had success in the clinical management of CINV, particularly in terms of vomiting and breakthrough antiemetics usage (i.e. CR). However, nausea experienced by cancer patients on emetogenic chemotherapies still remains less well-controlled than vomiting [33]. The recent re-classification of the $\mathrm{AC}$ regimen from being moder- ately-emetogenic to highly-emetogenic by the American Society of Clinical Oncology [34] suggests the need to improve the use of NK1-RAs, such as aprepitant, in CINV control. Furthermore, it was found that clinicians tend to underestimate the incidences of nausea [34], as well as overestimate the control of delayed CINV in patients on MECs and HECs without cisplatin [35]. As such, there is also imperative need for more research to be done in this area.

In the past year, published studies on NK1-RAs have strengthened the evidence on control of emesis, but not nausea $[27,33]$. There is a need for a paradigm shift in CINV research to be more focused on nausea control [36], since this symptom is distinct from vomiting and if it remains unresolved, can also impact patients' quality of life. Basic scientists should attempt to further understand the mechanisms of chemotherapy-induced nausea, while clinician-scientists should target optimal control of endpoints that incorporate good nausea control (i.e. $\mathrm{CP}$ or CC). While other medications (e.g. olanzapine-containing antiemetic regimens) have shown promise in nausea control compared to NK1-RAs [37], research in this area is still in its infancy and more substantiating studies are definitely needed.

Other areas in which CINV research should be expanded towards are in pharmacogenetics and individualization of antiemetic therapies for cancer patients. It is suggested that the suboptimal response to antiemetics could be due to the variability in genes which play a role in the pharmacokinetics and pharmacodynamics of antiemetic drugs [38]. According to a recent review [38], although there is sparse literature on the associations of pharmacogenetics with $5 \mathrm{HT}_{3}-\mathrm{RAs}$, there is none for the other antiemetics, including the NK1-RAs. While plasma aprepitant concentrations may be similar between Japanese and Westerners [39], other Asians (e.g. Chinese, Malays, Indians) and ethnic minorities may not experience equivalent therapeutic benefits since their pharmacogenetics may differ. The importance of this field will become more evident as newer NK1-RAs (e.g. rolapitant) [40] penetrate the market in the near future.

In addition, the advent of more advanced informatics technologies and computational strategies (e.g. virtual reality and 3D modelling) [41] can bring drug discovery and development to a whole new level through real-time physiological simulations and structure-activity modelling of therapeutic targets. The use of these newer technologies for computer simulations of future NK1-RAs and similar compounds are definitely attractive. Furthermore, newer care pathways involving e-health technologies (e.g. tele-monitoring) [42] and advanced computational analyses (e.g. machine learning) [43] have the potential to improve the pharmaceutical care of cancer patients thro- 
ugh reduction of chemotherapy-induced toxicities, such as CINV.

\section{Conclusion}

NK1-RAs are useful in significantly improving overall $\mathrm{CR}$ in various patient populations, regardless of the risk factors present. Thus, they could be a useful adjuvant in CINV control. Their introduction into antiemetic prophylaxis could make CINV less distressing for breast cancer patients, and improve their quality of life. However, we should bear in mind that they have not been shown to be effective in controlling nausea, or attaining $\mathrm{CC}$, the ideal CINV control endpoint. As healthcare providers continually modify their prescribing habits as they strive for the best CINV control in their patients, we hope this review may come in useful in aiding them to make better clinical decisions.

\section{REFERENCES}

[1] C. C. Sun, D. C. Bodurka, C. B. Weaver, et al., "Rankings and Symptom Assessments of Side Effects from Chemotherapy: Insights from Experienced Patients with Ovarian Cancer," Supportive Care in Cancer, Vol. 13, No. 4, 2005, pp. 219-227. doi:10.1007/s00520-004-0710-6

[2] A. Fabi, M. Barduagni, S. Lauro, et al., "Is Delayed Chemotherapy-Induced Emesis Well Managed in Oncological clinical Practice? An Observational Study," Supportive Care in Cancer, Vol. 11, No. 3, 2003, pp. 156-161. doi:10.1007/s00520-002-0427-3

[3] S. Grunberg, R. Deuson, P. Mavros, et al., "Incidence of Chemotherapy-Induced Nausea and Emesis after Modern Antiemetics: Perception versus Reality," Cancer, Vol. 100, No. 10, 2004, pp. 2261-2268. doi:10.1002/cncr.20230

[4] K. G. Reddy, R. J. Gralla and P. J. Hesketh, "Novel Neurokinin-1 Antagonists as Antiemetics for the Treatment of Chemotherapy-Induced Emesis," Supportive Cancer Therapy, Vol. 3, No. 3, 2006, pp. 140-142. doi:10.3816/SCT.2006.n.011

[5] M. Kris, P. Hesketh, M. Somerfield, et al., "American Society of Clinical Oncology Guideline for Antiemetics in Oncology: Update 2006," Journal of Clinical Oncology, Vol. 24, No. 18, 2006, pp. 2932-2947. doi:10.1200/JCO.2006.06.9591

[6] National Comprehensive Cancer Network, "NCCN Clinical Practice Guidelines in Oncology-Antiemesis (Version I.2012)," 2011.

http://www.nccn.org/professionals/physician_gls/PDF/ant iemesis.pdf

[7] R. J. Gralla, F. Roila, M. Tonato and J. Herrstedt, "MASCC/ ESMO Antiemetic Guideline 2011,” 2011. http://data.memberclicks.com/site/mascc/MASCC_Guide lines_English_2011.pdf

[8] R. Twycross and A. Wilcock, "Nausea and Vomiting," Symptom Management in Advanced Cancer, Radcliffe Me- dical Press, Oxford, 2002, pp. 104-110.

[9] S.-C. J. Yeung, C. P. Escalante and R. F. Gagel, "Medical Care of Cancer Patients," People's Medical Publishing House, USA, 2009.

[10] M. P. Curran and D. M. Robinson, "Aprepitant: A Review of Its Use in the Prevention of Nausea and Vomiting," Drugs, Vol. 69, No. 13, 2009, pp. 1853-1878. doi:10.2165/11203680-000000000-00000

[11] J. Herrstedt, W. Arpornwirat, A. Shaharyar, et al., "Phase III Trial of Casopitant, a Novel Neurokinin-1 Receptor Antagonist, for the Prevention of Nausea and Vomiting in Patients Receiving Moderately Emetogenic Chemotherapy," Journal of Clinical Oncology, Vol. 27, No. 32, 2009, pp. 5363-5369. doi:10.1200/JCO.2009.21.8511

[12] B. L. Rapoport, K. Jordan, J. A. Boice, et al., "Aprepitant for the Prevention of Chemotherapy-Induced Nausea and Vomiting Associated with a Broad Range of Moderately Emetogenic Chemotherapies and Tumor Types: A Randomized, Double-Blind Study," Supportive Care in Cancer, Vol. 18, No. 4, 2010, pp. 423-431. doi:10.1007/s00520-009-0680-9

[13] Health Promotion Board National Registry of Diseases Office, "Singapore Cancer Registry Interim Report: Trends in Cancer Incidence in Singapore 2004-2008," 2010. http://www.nrdo.gov.sg/uploadedFiles/NRDO/Publicatio ns/Cancer_Trends_Report0408_web_v2.pdf

[14] B. Fisher, S. Anderson, D. L. Wickerham, et al., "Increased Intensification and Total Dose of Cyclophosphamide in a Doxorubicin-Cyclophosphamide Regimen for the Treatment of Primary Breast Cancer: Findings from National Surgical Adjuvant Breast and Bowel Project B-22," Journal of Clinical Oncology, Vol. 15, No. 5, 1997, pp. 1858-1869.

[15] B. Fisher, A. M. Brown, N. V. Dimitrov, et al., "Two Months of Doxorubicin-Cyclophosphamide with and without Interval Reinduction Therapy Compared with 6 Months of Cyclophosphamide, Methotrexate and Fluorouracil in Positive-Node Breast Cancer Patients with Tamoxifen-Nonresponsive Tumors: Results from the National Surgical Adjuvant Breast and Bowel Project B-15," Journal of Clinical Oncology, Vol. 8, No. 9, 1990, pp. 1483-1496.

[16] J. M. Nabholtz, C. Falkson, D. Campos, et al., "Docetaxel and Doxorubicin Compared with Doxorubicin and Cyclophosphamide as First-Line Chemotherapy for Metastatic breast Cancer: Results of a Randomized, Multicenter, Phase III Trial," Journal of Clinical Oncology, Vol. 21, No. 6, 2003, pp. 968-975. doi:10.1200/JCO.2003.04.040

[17] T. Grote, J. Hajdenberg, A. Cartmell, et al., "Combination Therapy for Chemotherapy-Induced Nausea and Vomiting in Patients Receiving Moderately Emetogenic Chemotherapy: Palonosetron, Dexamethasone and Aprepitant," Journal of Supportive Oncology, Vol. 4, No. 8, 2006, pp. 403-408.

[18] W. Arpornwirat, I. Albert, V. L. Hansen, et al., "Phase 2 Trial Results with the Novel Neurokinin-1 Receptor Antagonist Casopitant in Combination with Ondansetron and Dexamethasone for the Prevention of Chemotherapy-In- 
duced Nausea and Vomiting in Cancer Patients Receiving Moderately Emetogenic Chemotherapy," Cancer, Vol. 115 , No. 24, 2009, pp. 5807-5816. doi: $10.1002 /$ encr. 24630

[19] J. Herrstedt, H. B. Muss, D. G. Warr, et al., "Efficacy and Tolerability of Aprepitant for the Prevention of Chemotherapy-Induced Nausea and Emesis over Multiple Cycles of Moderately Emetogenic Chemotherapy," Cancer, Vol. 104, No. 7, 2005, pp. 1548-1555. doi:10.1002/cncr.21343

[20] P. J. Hesketh, J. Younger, P. Sanz-Altamira, et al., "Aprepitant as Salvage Antiemetic Therapy in Breast Cancer Patients Receiving Doxorubicin and Cyclophosphamide," Supportive Care in Cancer, Vol. 17, No. 8, 2009, pp. 1065-1070. doi:10.1007/s00520-008-0545-7

[21] D. G. Warr, P. J. Hesketh, R. J. Gralla, et al., "Efficacy and Tolerability of Aprepitant for the Prevention of Chemotherapy-Induced Nausea and Vomiting in Patients with Breast Cancer after Moderately Emetogenic Chemotherapy," Journal of Clinical Oncology, Vol. 23, No. 12, 2005, pp. 2822-2830. doi:10.1200/JCO.2005.09.050

[22] W. Yeo, F. K. F. Mo, J. J. S. Suen, et al., "A Randomized study of Aprepitant, Ondansetron and Dexamethasone for Chemotherapy-Induced Nausea and Vomiting in Chinese Breast Cancer Patients Receving Moderately Emetogenic Chemotherapy," Breast Cancer Research and Treatment, Vol. 113, No. 3, 2009, pp. 529-535. doi:10.1007/s10549-008-9957-9

[23] M. S. Aapro and C. M. Walko, "Aprepitant: Drug-Drug Interactions in Perspective," Annals of Oncology, Vol. 21, No. 12,2010 , pp. 2316-2323. doi:10.1093/annonc/mdq149

[24] National Cancer Institute, "Common Terminology Criteria for Adverse Events v3.0 (CTCAE)," 2006. http://www.eortc.be/services/doc/ctc/ctcaev3.pdf

[25] R. Gralla, M. Lichinitser, S. Van der Vegt, et al., "Palonosetron Improved Prevention of Chemotherapy-Induced Nausea and Vomiting Following Moderately Emetogenic Chemotherapy: Results of a Double-Blind Randomized Phase III Trial Comparing Single Doses of Palonosetron with Ondansetron," Annals of Oncology, Vol. 14, No. 10, 2003, pp. 1570-1577. doi:10.1093/annonc/mdg417

[26] P. J. Hesketh, M. Aapro, J. C. Street and A. D. Carides, "Evaluation of Risk Factors Predictive of Nausea and Vomiting with Current Standard-of-Care Antiemetic Treatment: Analysis of Two Phase III Trials of Aprepitant in Patients Receiving Cisplatin-Based Chemotherapy," Supportive Care in Cancer, Vol. 18, No. 9, 2010, pp. 11711177. doi:10.1007/s00520-009-0737-9

[27] D. G. Warr, J. C. Street and A. D. Carides, "Evaluation of Risk Factors Predictive of Nausea and Vomiting with Current Standard-of-Care Antiemetic Treatment: Analysis of Phase 3 Trial of Aprepitant in Patients Receiving Adriamycin-Cyclophosphamide-Based Chemotherapy," Supportive Care in Cancer, Vol. 19, No. 6, 2011, pp. 807813. doi:10.1007/s00520-010-0899-5

[28] V. Shih, S. W. Hee and A. Chan, "Clinical Predictors of Chemotherapy-Induced Nausea and Vomiting in Breast Cancer Patients Receiving Adjuvant Doxorubicin and Cyclophosphamide," Annals of Pharmacotherapy, Vol. 43,

\section{No. 3, 2009, pp. 444-452. doi:10.1345/aph.1L437}

[29] J. K. Lamba, Y. S. Lin, E. G. Schuetz and K. E. Thummel, "Genetic Contribution to Variable Human CYP3A-Mediated Metabolism," Advanced Drug Delivery Reviews, Vol. 54, No. 10, 2002, pp. 1271-1294. doi:10.1016/S0169-409X(02)00066-2

[30] R. I. Sanchez, R. W. Wang, D. J. Newton, et al., "Cytochrome P450 3A4 Is the Major Enzyme Involved in the Metabolism of the Substance P Receptor Antagonist Aprepitant," Drug Metabolism and Disposition, Vol. 32, No. 11, 2004, pp. 1287-1292. doi:10.1124/dmd.104.000216

[31] J. F. Solus, B. J. Arietta, J. R. Harris, et al., "Genetic Variation in Eleven Phase I Drug Metabolism Genes in an Ethnically Diverse Population," Pharmacogenomics, Vol. 5, No. 7, 2004, pp. 895-931. doi:10.1517/14622416.5.7.895

[32] GlaxoSmithKline, "Withdrawal of Marketing Authorisation Application for Zunrisa (as Casopitant Mesilate), 50 $\mathrm{mg}$ and $150 \mathrm{mg}$ film coated tablets-EMEA/H/C/1040," 2009.

http://www.ema.europa.eu/docs/en_GB/document_library /Other/2010/01/WC500060224.pdf

[33] P. J. Hesketh and P. Sanz-Altamira, "Aprepitant, Dexamethasone, and Palonosetron in the Prevention of Doxorubicin/Cyclophosphamide-Induced Nausea and Vomiting," Supportive Care in Cancer, epub ahead of print, 2011. doi:10.1007/s00520-011-1312-8

[34] E. Basch, A. A. Prestrud, P. J. Hesketh, et al., "Antiemetics: American Society of Clinical Oncology Clinical Practice Guideline Update," Journal of Clinical Oncology, Vol. 29, No. 31, 2011, pp. 4189-4198. doi:10.1200/JCO.2010.34.4614

[35] M. Majem, M. E. Moreno, N. Calvo, et al., "Perception of Healthcare Providers versus Patient Reported Incidence of Chemotherapy-Induced Nausea and Vomiting after the Addition of NK-1 Receptor Antagonists," Supportive Care in Cancer, Vol. 19, No. 12, 2011, pp. 1983-1990. doi:10.1007/s00520-010-1042-3

[36] S. M. Grunberg, D. Warr, R. J. Gralla, et al., "Evaluation of New Antiemetic Agents and Definition of Antineoplastic Agent Emetogenicity-State of the Art," Supportive Care in Cancer, Vol. 19, No. Supplement 1, 2011, pp. S43-S47. doi:10.1007/s00520-010-1003-X

[37] R. M. Navari, S. E. Gray and A. C. Kerr, "Olanzapine versus Aprepitant for the Prevention of ChemotherapyInduced Nausea and Vomiting: A Randomized Phase III Trial," Journal of Supportive Oncology, Vol. 9, No. 5, 2011, pp. 188-195. doi:10.1016/j.suponc.2011.05.002

[38] D. A. Perwitasari, H. Gelderblom, J. Atthobari, et al., "Anti-emetic Drugs in Oncology: Pharmacology and Individualization by Pharmacogenetics," International Journal of Clinical Pharmacy, Vol. 33, No. 1, 2011, pp. 33-43. doi:10.1007/s11096-010-9454-1

[39] T. Takahashi, Y. Nakamura, A. Tsuya, et al., "Pharmacokinetics of Aprepitant and Dexamethasone after Administration of Chemotherapeutic Agents and Effects of Plasma Substance P Concentration on Chemotherapy-In- 
duced Nausea and Vomiting in Japanese Cancer Patients," Cancer Chemotherapy and Pharmacology, Vol. 68, No. 3, 2011, pp. 653-659. doi:10.1007/s00280-010-1519-2

[40] T. J. Gan, J. Gu, N. Singla, et al., "Rolapitant for the Prevention of Postoperative Nausea and Vomiting: A Prospective, Double-Blinded, Placebo-Controlled Randomized trial," Anesthesia and Analgesia, Vol. 112, No. 4, 2011, pp. 804-812. doi:10.1213/ANE.0b013e31820886c3

[41] K. Dhanachandra Singh, M. Karthikeyan, P. Kirubakaran and S. Nagamani, "Pharmacophore Filtering and 3D-QSAR in the Discovery of New JAK2 Inhibitors," Journal of Molecular Graphics and Modelling, Vol. 30, 2011, pp.
186-197. doi:10.1016/j.jmgm.2011.07.004

[42] A. Weaver, A. M. Young, J. Rowntree, et al., "Application of Mobile Phone Technology for Managing Chemotherapy-Associated Side-Effects," Annals of Oncology, Vol. 18, No. 11, 2007, pp. 1887-1892.

doi:10.1093/annonc/mdm354

[43] K. Y.-L. Yap, X. R. Yak, V. Shih, W. K. Chui and A. Chan, "Application of Unsupervised Learning in Clinical Oncology Practice-Exploring Anxiety Characteristics in Chemotherapy-Induced Nausea and Vomiting through Principal Variables," Journal of Computing, Vol. 2, No. 7, 2010, pp. 163-171. 